\title{
Acquiring Applicable Common Sense Knowledge from the Web
}

\author{
Hansen A. Schwartz and Fernando Gomez \\ School of Electrical Engineering and Computer Science \\ University of Central Florida \\ Orlando, FL 32816, USA \\ \{hschwartz, gomez\}@cs.ucf.edu
}

\begin{abstract}
In this paper, a framework for acquiring common sense knowledge from the Web is presented. Common sense knowledge includes information about the world that humans use in their everyday lives. To acquire this knowledge, relationships between nouns are retrieved by using search phrases with automatically filled constituents. Through empirical analysis of the acquired nouns over WordNet, probabilities are produced for relationships between a concept and a word rather than between two words. A specific goal of our acquisition method is to acquire knowledge that can be successfully applied to NLP problems. We test the validity of the acquired knowledge by means of an application to the problem of word sense disambiguation. Results show that the knowledge can be used to improve the accuracy of a state of the art unsupervised disambiguation system.
\end{abstract}

\section{Introduction}

Common sense knowledge (CSK) is the knowledge we use in everyday life without necessarily being aware of it. Panton et al. (2006) of the Cyc project, define common sense as "the knowledge that every person assumes his neighbors also possess". Although the term common sense may be understood as a process such as reasoning, we are referring only to knowledge. It is CSK that tells us keys are kept in one's pocket and keys are used to open a door, but CSK does not hold that keys are kept in a kitchen sink or that keys are used to turn on a microwave, although all are possible.

To show the need for this information more clearly we provide a couple sentences:
She put the batter in the refrigerator.

He ate the apple in the refrigerator.

In (1), we are dealing with lexical ambiguity. There is little doubt for us to determine just what the "batter" is (food/substance used in baking). However, a computer must determine that it is not someone who swings a bat in baseball that is being put into a refrigerator, although it is entirely possible to do (depending on the size of the refrigerator). This demonstrates how CSK can be useful in solving word sense disambiguation. We know it is common for food to be found in a refrigerator and so we easily resolve batter as a food/substance rather than a person.

CSK can also help to solve syntactic ambiguity. The problem of prepositional phrase attachment occurs in sentences similar to (2). In this case, it is difficult for a computer to determine if "he" is in the refrigerator eating an apple or if the "apple" which he ate was in the refrigerator. Like the previous example, the knowledge that food is commonly found in a refrigerator and people are not, leads us to understand that "in the refrigerator" should be attached to the noun phrase "the apple" and not as a modifier of the verb phrase "ate".

Unfortunately, there are not many sources of CSK readily available for use in computer algorithms. Those sets of knowledge that are available, such as the CYC project (Lenat, 1995) or ConceptNet (Liu and Singh, 2004) rely on manually provided or crafted data. Our aim is to develop an automatic approach to acquire $C S K^{1}$ by turning to the vast amount of unannotated text that is available on the Web. In turn, we present a method to automatically retrieve and analyze phrases from the Web.

\footnotetext{
${ }^{1}$ data available at: http://eecs.ucf.edu/ ${ }^{2}$ hschwartz/CSK/
} 
We employ the use of a syntactic parser to accurately match syntactic patterns of phrases acquired from the Web. The data is analyzed over WordNet (Miller et al., 1993) in order to induce knowledge about word senses or concepts rather than words. Finally, we evaluate whether the knowledge by applying it to the problem of word sense disambiguation.

\section{Background}

The particular type of $C S K$ that we experiment with in this paper is described formally as follows:

A relationship, $e 1 \mathbf{R} e 2$, exists between entities $e 1$ and $e 2$ if one finds " $e 1$ is $\mathbf{R} e 2$."

Some examples include: "a cup is on a table" and "food is in a refrigerator", which would result in relationships: cupontable and foodinrefrigerator. The next section attempts to make the relationship more clear, as we provide a brief linguistic background of prepositions and relationships.

\subsection{Prepositions and Relationships}

Prepositions state a relationship between two entities (Quirk et al., 1985). One of the entities is typically a constituent of the sentence while the other is the complement to the preposition. For example, consider the relationship between 'furniture' and 'house' in the following sentences:

\section{The furniture is...}

\section{.... at the house. \\ ...on the house. \\ ...in the house.}

'The furniture' is the subject of the sentence, while 'the house' is a prepositional complement. Notice that the meaning is different for each sentence depending on the actual preposition ('at', 'on', or 'in'), and thus furniture relates to house in three different ways. Although each relationship between furniture and house is possible, only one would be considered CSK to most people: furnitureinhouse.

We focus on prepositions which indicate a positive spacial relationship given by Quirk et al. (1985). There are three types of such relationships: "at a point", "on a line or surface", and "in an area or volume". In particular, we concentrate on the 1 to 3 dimensional relationships given in Table 1, denoted on and in throughout the paper. At, the 0 dimensional relationship, occurred far less frequently. The

\begin{tabular}{|c|c|l|}
\hline dims & description & prepositions \\
\hline 1 or 2 & on surface or line & $\begin{array}{l}\text { on, onto, atop, upon, } \\
\text { on top of, down on }\end{array}$ \\
\hline 2 or 3 & in area or volume & $\begin{array}{l}\text { in, into, inside, } \\
\text { within, inside of }\end{array}$ \\
\hline
\end{tabular}

Table 1: Spatial dimensions (dims) and corresponding prepositions.

sentences below exemplify each of the 1 to 3 dimensional relationships:

on surface The keyboard is on the table.

on line The beach is on US 1.

in area The bank is in New York.

in volume The vegetables are in the bowl.

\subsection{Related Work}

As a prevalent source of lexical knowledge, dictionary definitions may be regarded as common sense. However, some definitions may be considered expert knowledge rather than CSK. The scope of definitions certainly do not provide all necessary information (such as keys are commonly kept in one's pocket). We examine WordNet in particular because the hypernym relation has been developed extensively for nouns. The noun ontology is used in our work to help induce relationships involving concepts (senses of nouns) rather than just among words. This notion of inducing CSK among concepts, rather than words, is a key difference between our work and similar research.

The work on VerbOcean is similar to our research in the use of the Web for acquiring relationships (Chklovski and Pantel, 2004). They used patterns of phrases in order to search the Web for semantic relations among verbs. The knowledge they acquire falls into the category of $C S K$, but the specific relationships are different than ours in that they are among verb word forms and senses are not resolved.

ConceptNet was created based on the OpenMind Commonsense project (Liu and Singh, 2004). The project acquired knowledge through an interface on the Web by having users play games and answer questions about words. A contribution of ConceptNet is that it has a wide range of relations. While WordNet provides connections between concepts (senses of words), ConceptNet only provides relationships between word forms. 


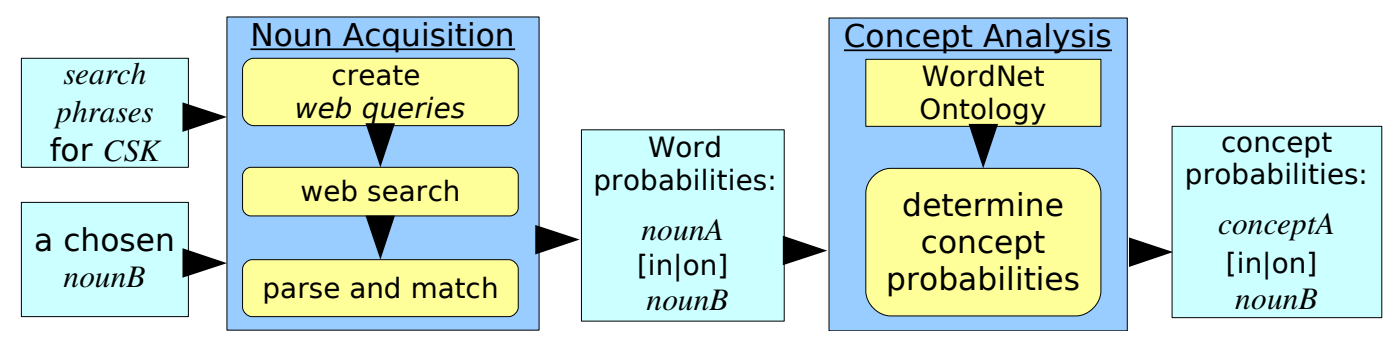

Figure 1: The overall common sense knowledge acquisition framework under the assumption that one is acquiring concepts (WordNet synsets) in a relationship with a given nounB (word).

A project in progress for over twenty years, CYC has been acquiring common sense knowledge about everyday objects and actions stored in $10^{6}$ axioms (Lenat, 1995). The axioms, handcrafted by workers at CYCcorp, represent knowledge rooted in propositions. There are three layers of information: the first two, access and physical, contain meta data, while the third, logical layer, stores high level implicit meanings. Only a portion of CYC is available to the public.

Our method for acquiring knowledge is somewhat similar to that of (Hearst, 1992). Patterns are built manually. However, we do not use our manually constructed patterns (referred to as search phrases) to query the Web. Instead the search phrases are abstract patterns that are used to automatically generate more specific web queries by filling constituents based on lists of words.

The SemEval-2007 Task 4 presents a good overview of work in noun-noun relationships (Girju et al., 2007). Our work is related in that the relationships we acquire are between nominals, and in order to build their corpus Girju et al. queried the web with patterns like that of Hearst's work (Hearst, 1992). The SemEval task was to choose or classify relationships, rather than acquire and apply relationships. Additionally, the relationship classes they use are not necessarily within the scope of common sense knowledge.

Similar to our research, in (Agirre et al., 2001) knowledge is acquired about WordNet concepts. They find topics signatures, sets of related words, based on data from the Web and use them for word sense disambiguation. However, the type of relationship between words of a topic signature and the WordNet concept is not made explicit, and the au- thors find the topic signatures are not very effective for word sense disambiguation.

Finally, we note one approach to using the Web for NLP applications is to acquire knowledge on the fly. Previous work has approached solutions to word sense disambiguation by acquiring words or phrases directly based on the sentences or words being disambiguated (Martinez et al., 2006; Schwartz and Gomez, 2008). These methods dynamically acquire the data at runtime, rather than automatically create a common sense database of relations that is readily available. Additionally, in our current approach, we are able to acquire explicit $C S K$ relationships.

\section{Common Sense Acquisition}

The two major phases of our framework, "Noun Acquisition" and "Concept Analysis", are outlined in Figure 1 and described within this section.

\subsection{Noun Acquisition}

The first step of our method is to acquire nouns (as words) from the Web which are in a relationship with other nouns. A Web search is performed in order to retrieve samples of text matching a web query created from a search phrase for the relationship. Each sample is syntactically parsed to verify a match with the corresponding web query, and the noun(s) filling a missing constituent of the parse are recorded.

The framework itself is very flexible, and it can handle the acquisition of words from other parts of speech. However, to be clear, we focus the explanation on the use of the framework to acquire specific types of relationships between nouns. Below we describe the procedures in more detail. 


\subsubsection{Creating Web Queries}

Web queries are created semi-automatically by defining these parameters of a search phrase:

nounA the first noun phrase

nounB the second noun phrase

prep preposition, if any, used in the phrase

verb verb, if any, used in the phrase.

Table 2 lists all of the search phrases we use, one of which we use as an example throughout this section:

\section{place nounA prep nounB}

The verb, "place" in this case, is statically defined as part of the search phrase.

Prepositions were chosen to describe the type of relationship we were seeking to acquire as described in the background section. We limited ourselves to the "on" and "in" relationships since these were the most common.

on $=($ on, onto, atop, upon, on top of, down on $)$

in $=($ in, into, inside, within, inside of $)$

When noun parameters are provided, determiners or possessive pronouns selected from the list below are included. This provides greater accuracy in our search results.

det $=($ the, a/an, this, that, my, your, his, her $)$

Finally, the undefined parameters are replaced with a '*'. Below is a web query created from our search phrase where nounB is 'refrigerator', prep is 'in', det is 'the', and nounA is undefined:

place $*$ in the refrigerator

\subsubsection{Searching the Web}

Given a nounB, The search algorithm can be summarized through the pseudocode below.

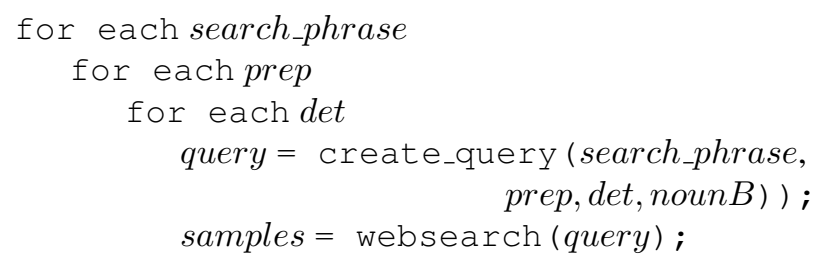

The searches were carried out through the Google Search $\mathrm{API}^{2}$, or the Yahoo! Search Web Services ${ }^{3}$. Each search phrase, listed in Table 2, was run until a maximum of 2000 results were returned. Duplicate samples were removed to reduce the effects of websites replicating the text of one another.

\footnotetext{
${ }^{2}$ no longer supported by Google

${ }^{3} \mathrm{http}: / /$ developer.yahoo.com/search/
}

\begin{tabular}{|c|l|c|}
\hline relation & search phrase & voice \\
\hline on, in & $\begin{array}{l}\text { nounA is located prep nounB } \\
\text { nounA is found prep nounB } \\
\text { nounA is situated prep nounB }\end{array}$ & passive \\
\hline on, in & $\begin{array}{l}\text { nounA is prep nounB } \\
\text { put nounA prep nounB } \\
\text { place nounA prep nounB } \\
\text { lay nounA prep nounB } \\
\text { set nounA prep nounB } \\
\text { locate nounA prep nounB } \\
\text { position nounA prep nounB }\end{array}$ & active \\
\hline on & $\begin{array}{l}\text { hang nounA prep nounB } \\
\text { mount nounA prep nounB } \\
\text { attach nounA prep nounB }\end{array}$ & active \\
\hline
\end{tabular}

Table 2: Search phrases and relationships used for acquisition of CSK.

\subsubsection{Parse and Match}

The results we want to achieve in this step should describe a relationship:

$$
\text { nounA is [in } \mid \text { on] nounB }
$$

We use Charniak's parser (Charniak, 2000) on both the web query and the results returned from the web in order to ensure accuracy. To demonstrate this process, we extend our example, "place $*$ in the refrigerator".

First, we get a parse with * (nounA) represented as 'something'.

(VP (VB place)

(NP (NN something))

(PP (IN in) (NP (DT the) (NN refrigerator))))

We now know the constituent(s) which replace '(NN something)' will be our nounA. For example, in the following parse 'batter' is resolved as nounA.

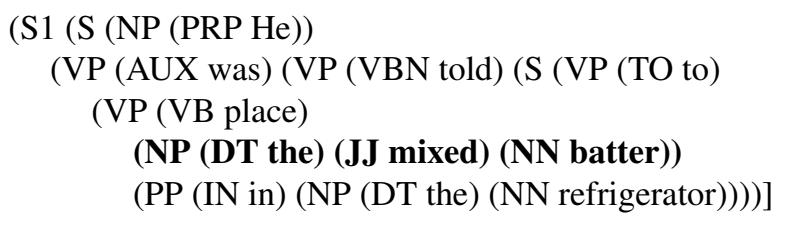

The head noun of the matching phrase is determined, which is 'batter' in the phrase '(DT the) (JJ mixed) (NN batter)'. Words are only recorded if they are present as a noun in WordNet. If the noun phrase contains a compound noun found in WordNet, then the compound noun is recorded instead.

The parse also helps to eliminate bad results. For the following sentence, the verb phrase does not 
match the parse of the web query due to an extra PP, and therefore we do not pull out "for several hours" as nounA.

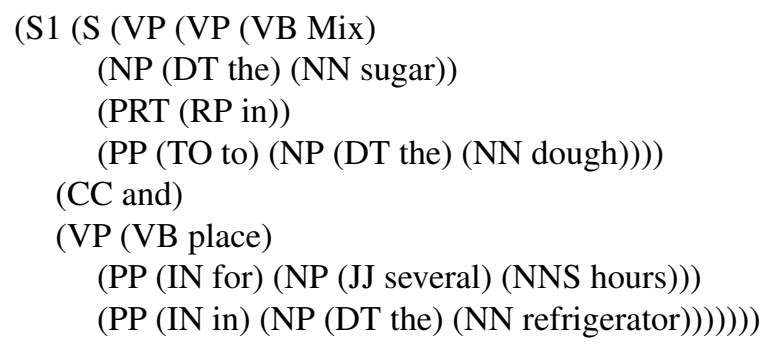

One may note that this malformed sentence is communicating that 'dough' is placed in the refrigerator, but the method does not handle this.

At the end of the noun acquisition phase, we are left with frequency counts of nouns being retrieved from a context matching the syntactic structure of a web query. This can easily be represented as the probability of a noun, $n A$, being returned to a query for the relationship, $\mathbf{R}$, with noun $n B$.

$$
p_{w}(n A, \mathbf{R}, n B)
$$

This value along with the other steps we have gone over are stored in a MySQL relational database ${ }^{4}$. One could trace a relationship probability between nouns back to the web results which were matched to a web query, and even determine the abstract search phrase which produced the web query.

\subsection{Concept Analysis}

A focus of this work is on going beyond relationships between words. We would like to acquire knowledge about specific concepts in WordNet. In particular, we are trying to induce:

$$
\text { conceptA is [in } \mid \text { on] nounB. }
$$

where conceptA is a concept in WordNet (such as a sense of nounA), and nounB remains simply a word.

For the analysis, we rely on the vast amount of nouns we are able to acquire in order to create probabilities for relationships of conceptARnounB. To get a grasp of the idea in general, consider 'table' as a nounB of interest. By examining all possible hypernyms of all senses of each noun $A$ one will find it is common for abstract entities to be "in a table" (i.e. data in a table), artifacts to be "on a table" (i.e.

\footnotetext{
${ }^{4}$ http://www.mysql.com
}

cup on a table), and physical things (including living things) to be "at a table" (i.e. the employees at the table). The same idea could be applied in reverse if one acquires knowledge for a set of nounAs. However, this paper only focuses on acquiring knowledge for the nounB constituent in a search phrase.

To begin with, one should note that concepts in WordNet are represented as synsets. A synset is a group of word-senses that have the same meaning. For example, (batter-1, hitter-1, slugger-1, batsman-1) is a synset with the meaning "(baseball) a ballplayer who is batting". We use WordNet version 3.0 in order to take advantage of the latest updates and corrections to the noun ontology. Since a word has multiple senses, we represent the probability that a word-sense, $n A s$, resulted from a query for a relationship, $\mathbf{R}$ with $n o u n B$ as:

$$
p_{n s}(n A s, \mathbf{R}, n B)=\frac{p_{w}(\text { lemma }(n A s), \mathbf{R}, n B)}{\text { senses }(\text { lemma }(n A s))}
$$

where senses returns the number of senses of the word (lemma) within the word-sense $n A s$. We can then extend the probability to apply to a synset, syns, as:

$$
p_{\text {syn }}(\text { syns }, \mathbf{R}, n B)=\sum_{n A s \in \text { syns }} p_{n s}(n A s, \mathbf{R}, n B)
$$

Finally, we define a recursive function based on the idea that a concept subsumes all concepts below it (hyponyms) in the WordNet ontology:

$$
\begin{aligned}
P_{c}(c A, \mathbf{R}, n B)= & p_{\text {syn }}(\operatorname{syns}(c A), \mathbf{R}, n B) \\
+ & \sum_{h \in \operatorname{hypos}(c A)} P_{c}(h, \mathbf{R}, n B)
\end{aligned}
$$

where $c A$ is a concept/node in WordNet, syns returns the synset which represents the concept, and hypos returns the set of all direct hyponyms within the WordNet ontology. For example, (money-3) is a (currency-1), so $P_{c}$ (currency-1, $\left.\mathbf{R}, n B\right)$ receives $p_{\text {syn }}(($ money-3), $\mathbf{R}, n B)$ among others. This type of calculation over WordNet follows much like that of Resnik's (1999) information-content calculation. Note that the function no longer recurs when reaching a concept with no hyponyms and that $P_{c}($ entity- $1, \mathbf{R}, n B)$ is always 1 (entity- 1 is the root node). $P_{c}$ now represents a probability for the relationship: conceptARnounB. 


\begin{tabular}{|ll|ll|}
\hline nounB & \#nounAs & nounB & \#nounAs \\
\hline basket & 3300 & boat & 2787 \\
bookcase & 260 & bottle & 4742 \\
bowl & 5252 & cabin & 720 \\
cabinet & 1474 & canoe & 163 \\
car & 5534 & ceiling & 1187 \\
city & 1432 & desk & 4770 \\
drawer & 1638 & dresser & 698 \\
floor & 2850 & house & 4627 \\
jar & 4462 & kitchen & 2948 \\
pocket & 4771 & refrigerator & 2897 \\
road & 5493 & room & 5023 \\
shelf & 2581 & ship & 1469 \\
sink & 296 & sofa & 509 \\
table & 5312 & truck & 528 \\
van & 301 & wall & 2285 \\
\hline
\end{tabular}

Table 3: List of nouns which fill the nounB constituent in a search phrase, and the corresponding occurrences of noun $A$ s acquired for each.

\section{Evaluation}

Our evaluation focuses on the applicability of the acquired CSK. We acquired relationships for the 30 nouns listed in Table 3. These nouns represent all possible words to fill the nounB constituent of a search phrase. The corresponding \#nounAs indicates the number of nounAs that were acquired from the Web for each nounB. For example, 4771 nounAs were acquired for 'pocket'. This means 4771 results from the web matched the parse of a web query for 'pocket' and contained a nounA in WordNet (keeping in mind duplicates Web text were removed).

Delving deeper into our example, below are the top 20 nounAs found for the relationship nounAinpocket.

money, hand, cash, firework, something, dollar, ball, hands, key, coin, pedometer, card, battery, item, phone, penny, music, buck, implant, wallet

As described in the concept analysis section, occurrences of each nounA for a given nounB lead to $p_{w}$ values, which in turn are used to produce $P_{c}$ values for concepts in WordNet. The application of CSK utilizes these probabilities rather than simply lists of words or even lists of concepts. However, challenges were encountered during the noun acquisition step before the probabilities were produced.

Many challenges of the noun acquisition step were overcome through the use of a parser. For example, phrases such as "Palestine is on the road to becoming..." could be eliminated since the parser marks the prepositional phrase "to becoming" as being attached to "the road". Thus, the parse of the web sample does not match the parse of the web query used to acquire it. Other times, noun-noun relationships were common simply because many web pages seem to copy the text of others. This problem was handled through the elimination of duplicate text samples from the Web. In the end, only about one in four results from the Web were actually used. Numbers in Table 3 reflect the result of these eliminations.

Some issues of the acquisition step were not directly addressed in this paper. A domain may tend to be more prevalent on the Internet and skew the CSK, such as fireworkinpocket. Another example, babyinbasket was very common due to biblical references. Fictional works and metaphors also provided uncommon relationships dispersed within the results. Additionally, the parser makes mistakes. It was the hope that the concept analysis step would help to mitigate some noise from these problems. A final issue was the bottleneck of limited queries per day by the search engines, which restricted us to testing on only the 30 nouns listed.

\subsection{Disambiguation System}

The CSK is not intended to be used by itself for disambiguation. It would be far from accurate to assume the sense of a noun can be disambiguated simply by observing its relationship with one other noun in the sentence. For example, one of the test sentences incorporated the relationship noteinpocket. Multiple senses of note are likely to be found in a pocket (i.e. the senses referring to "a brief written record", "a short personal letter", or "a piece of paper money"). In other cases, a relationship may not be found for any sense of a target word. Therefore, our knowledge is intended to be used as a reference, consulted by a disambiguation system.

We integrate our knowledge into a state of the art "all-words" word sense disambiguation algorithm. These algorithms are considered unsupervised or 
minimally supervised, because they do not require specific training data that is designed for instances of words in the testing data. In other words, these systems are designed to handle any word they come across. Our knowledge can supplement such a system, because the data can be acquired automatically for an unlimited number of nouns, assuming limitless web query restrictions.

The basis of our disambiguation system is the publicly available GWSD system (Sinha and Mihalcea, 2007). Sinha and Mihalcea report higher results on the Senseval-2 and Senseval-3 datasets than any of the participating unsupervised system. Additionally, GWSD is compatible with WordNet 3.0 and its output made it easy to integrate our knowledge. Sense predictions from four different graph metrics are produced, and we are able to incorporate our knowledge as another prediction within a voting scheme.

Considering the role of our knowledge as a reference, in some cases we would like the CSK to suggest multiple senses and in others none. For each target noun instance in the corpus, we lookup the $P_{c}(c, \mathbf{R}, n B)$ value, where $c$ is the WordNet concept that corresponds to a sense of the target noun. We choose $n B$ by matching the phrase "in|on $\operatorname{det} n B$ " within the sentence. The system suggests all senses with a $P_{c}$ value greater than 0.75 of the maximum $P_{c}$ value over all senses. If no senses have a $P_{c}$ value then no senses are suggested.

During voting, tallies of predictions and suggestions are taken for each sense of a noun. Ties are broken by choosing the lowest sense number among all those involved in the tie. Note that this is different than choosing the most frequent sense (i.e. the lowest sense number from all senses), in that only the top predicted senses are considered. This same type of voting is used with and without the CSK suggestions.

\subsection{Experimental Corpus}

A goal of our work was to acquire data which could be applied to NLP problems. We focus particularly on the difficult problem of word sense disambiguation. Due to the lack of sense tagged data, we were unable to find an annotated corpus with instances of all the nouns in Table 3 as prepositional complements. This was not surprising considering one of the reasons that minimally supervised approaches have become more popular is that they do not require hand-tagged training data (Mihalcea, 2002; Diab, 2004; McCarthy et al., 2004).

We created a corpus from sentences in Wikipedia which contained the phrase "in|on det lemma", where det is a determiner or possessive pronoun, lemma is a noun from Table 3, and in $\mid$ on is a preposition for either relationship described earlier. Below we have provided an example from our corpus where the knowledge from 'pocket' can be applied to disambiguate 'key'.

Now Tony's key to the flat is in the pocket of his raincoat, so on returning to his flat some time later he realizes that he cannot get inside.

The corpus ${ }^{5}$ contained a total of 342 sentences, with one target noun annotated per sentence. The target nouns were selected to potentially fill the nounA constituent in the relationship nounA $\mathbf{R}$ nounB, and they were assigned all appropriate WordNet 3.0 senses. Considering the finegrained nature of WordNet (Ide and Wilks, 2006), $26.3 \%$ of the instances were annotated with multiple senses. We also restricted the corpus to only include polysemous nouns, or nouns which had an additional sense beyond the senses assigned to it.

Inter-annotator agreement was used to validate the corpus. Because the corpus was built by an author of the work, we asked a non-author to reannotate the corpus without knowledge of the original annotations. This second annotator was told to choose all appropriate senses just as did the original annotator. Agreement was calculated as:

$$
\text { agree }=\left(\sum_{i \in C} \frac{\left|S 1_{i} \cap S 2_{i}\right|}{\left|S 1_{i} \cup S 2_{i}\right|}\right) \div 342
$$

where $S 1$ and $S 2$ are the two sets of sense annotations, and $i$ is an instance of the corpus, $C$.

The agreement and other data concerning corpus annotation can be found in Table 4. As a point of comparison, the Senseval 3 all-words task had a 75\% agreement on nouns (Snyder and Palmer, 2004). A second evaluation of agreement was also done. The non-author annotations were treated as if they came

\footnotetext{
${ }^{5}$ available at: http://eecs.ucf.edu/ ${ }^{\text {hschwartz/CSK/ }}$
} 


\begin{tabular}{|c|c|c|ccc|}
\hline & insts & agree & F1 $_{h}$ & F1 $_{\text {rnd }}$ & F1 $_{M F S}$ \\
\hline on & 131 & 79.9 & 84.7 & 28.2 & 71.0 \\
in & 211 & 80.8 & 91.9 & 27.2 & 67.8 \\
\hline both & 342 & 80.5 & 89.2 & 27.6 & 69.0 \\
\hline
\end{tabular}

Table 4: Experimental corpus data for each relationship (on, in). insts: number of annotated instances; agree: inter-annotator agreement \%; F1 values (precision $=$ recall): $h$ : human annotation, $r n d$ : random baseline, $M F S$ : most frequent sense baseline.

\begin{tabular}{|c|cc|cc|}
\hline & \multicolumn{2}{|c|}{ without $\boldsymbol{C S K}$} & \multicolumn{2}{c|}{ with $\boldsymbol{C S K}$} \\
& F1 $_{\text {all }}$ & F1 $_{\text {indeg }}$ & F1 $_{\text {all }}$ & F1 $_{\text {indeg }}$ \\
\hline on & 62.6 & 63.4 & 64.9 & 67.2 \\
in & 68.7 & 69.7 & 71.6 & 72.5 \\
\hline both & 66.4 & $\mathbf{6 7 . 3}$ & 69.0 & $\mathbf{7 0 . 5}$ \\
\hline ties & 37 & 0 & 66 & 72 \\
\hline
\end{tabular}

Table 5: F1 values (precision = recall) on our experimental corpus with and without $C S K$. F1 $\mathbf{1}_{\text {all }}$ : using all 4 graph metrics; $\mathbf{F} 1_{\text {indeg }}$ : using only the indegree metric; ties: number of instances where tie votes occurred.

from a disambiguation system in order to get a human upper-bound of performance. Just as the automatic system handled tie votes, when one word had multiple sense annotations, the annotation with the lowest sense number was used. This performance upper-bound is shown as $\mathbf{F} \mathbf{1}_{h}$ in Table 4.

\subsection{Results}

Our disambiguation results are presented in Table 5. We found that, in all cases, including CSK improved results. It turned out that $54.7 \%$ of the noun instances received at least one suggestion from the CSK, and $24.5 \%$ of the instances received multiple suggestions. It is not clear why the on results were slightly below that for $\boldsymbol{i n}$. We suspect the on portion of the corpus was slightly more difficult because the human annotation $\left(\mathbf{F} \mathbf{1}_{h}\right)$ found a similar phenomenon.

One observation we made when setting up the test was that the indegree metric alone performed slightly better than using the votes of all four metrics. This was not surprising considering Sinha and Mihalcea found the indegree metric by itself to perform only slightly below a combination of metrics on the senseval data (Sinha and Mihalcea, 2007).
Therefore, Table 5 also reports the use of the indegree metric by itself or with $C S K, \mathrm{~F} 1_{\text {indeg }}$. In these cases we saw the greatest improvements of using CSK, producing an an error reduction of about $4.5 \%$ and outperforming the $\mathrm{F} 1_{M F S}$ value.

Several additional experiments were performed. Note that even during ties, the chosen sense was taken from the predictions and suggestions. When we instead incorporated an MFS backoff strategy for ties, our top results for $\mathbf{F} \mathbf{1}_{\text {indeg }}$ with $C S K$ dropped to 70.2. We also ran a precision test with no predictions made for tie votes, and found a precision of $71.9 \%$ on the 270 instances that did not have a tie for top votes (this also used the indegree metric with $C S K$ ). All results supported our goal of acquiring $C S K$ that was applicable to word sense disambiguation.

\section{Conclusion}

We found our acquired $C S K$ to be useful when incorporated into a word sense disambiguation system, finding an error reduction of around $4.5 \%$ for top results. Relationships between nouns were acquired from the Web through a unique search method of filling constituents in a search phrase. Samples returned from the Web were restricted by a requirement to match the syntactic parse of a web query. The resulting data was analyzed over WordNet to produce probabilities of relationships in the form of conceptARnounB, where conceptA is a concept in WordNet rather than an ambiguous noun.

In our effort to validate the knowledge through application, many steps along the way were left open for future investigations. First, there is a need to exhaustively search for CSK of all nouns and to acquire other forms of CSK. With this improvement CSK could be tested on a standard corpus, rather than a corpus focused on select nouns and prepositional phrases. Looking into acquisition improvements, a study of the effectiveness of the parse would be beneficial. Finally, the applicability of the knowledge may be increased through a more complex concept analysis or utilizing a more advanced voting scheme.

\section{Acknowledgement}

This research was supported by the NASA Engineering and Safety Center under Grant/Cooperative Agreement NNX08AJ98A. 


\section{References}

Eneko Agirre, Olatz Ansa, and David Martinez. 2001. Enriching wordnet concepts with topic signatures. In In Proceedings of the NAACL workshop on WordNet and Other Lexical Resources: Applications, Extensions and Customizations, Pittsburg, USA.

Eugene Charniak. 2000. A maximum-entropy-inspired parser. In Proceedings of the first conference on North American chapter of the Association for Computational Linguistics, pages 132-139, San Francisco, CA, USA. Morgan Kaufmann Publishers Inc.

Timothy Chklovski and Patrick Pantel. 2004. Verbocean: Mining the web for fine-grained semantic verb relations. In Proceedings of Conference on Empirical Methods in Natural Language Processing (EMNLP04), Barcelona, Spain.

Mona Diab. 2004. Relieving the data acquisition bottleneck in word sense disambiguation. In Proceedings of the 42nd Annual Meeting of the Association for Computational Linguistics (ACL'04), pages 303-310.

Roxana Girju, Preslav Nakov, Vivi Nastase, Stan Szpakowicz, Peter Turney, and Deniz Yuret. 2007. Semeval-2007 task 04: Classification of semantic relations between nominals. In Proceedings of SemEval2007, pages 13-18, Prague, Czech Republic, June. Association for Computational Linguistics.

Marti A. Hearst. 1992. Automatic acquisition of hyponyms from large text corpora. In In Proceedings of the 14th International Conference on Computational Linguistics (COLING-92), pages 539-545.

Nancy Ide and Yorick Wilks, 2006. Word Sense Disambiguation: Algorithms And Applications, chapter 3: Making Sense About Sense. Springer.

Douglas B. Lenat. 1995. CYC: a large-scale investment in knowledge infrastructure. Communications of the ACM, 38(11):33-38.

H. Liu and P Singh. 2004. Conceptnet: A practical commonsense reasoning toolkit. BT Technology Journal, 22:211-226.

David Martinez, Eneko Agirre, and Xinglong Wang. 2006. Word relatives in context for word sense disambiguation. In Proceedings of the 2006 Australasian Language Technology Workshop, pages 42-50.

Diana McCarthy, Rob Koeling, Julie Weeds, and John Carroll. 2004. Finding predominant word senses in untagged text. In Proceedings of the 42nd Meeting of the Association for Computational Linguistics, pages 279-286, Barcelona, Spain, July. Association for Computational Linguistics.

Rada Mihalcea. 2002. Bootstrapping large sense tagged corpora. In Proceedings of the 3rd International Conference on Languages Resources and Evaluations LREC 2002, Las Palmas, Spain, May.
George Miller, R. Beckwith, Christiane Fellbaum, D. Gross, and K. Miller. 1993. Five papers on wordnet. Technical report, Princeton University.

Kathy Panton, Cynthia Matuszek, Douglas Lenat, Dave Schneider, Michael Witbrock, Nick Siegel, and Blake Shepard. 2006. Common sense reasoning : From cyc to intelligent assistant. In Y. Cai and J. Abascal, editors, Ambient Intelligence in Everyday Life, pages 131.

Randolph Quirk, Sidney Greenbaum, Geoffrey Leech, and Jan Svartvik. 1985. A Comprehensive Grammaer of the English Language. Longman.

Philip Resnik. 1999. Semantic similarity in a taxonomy: An information-based measure and its application to problems of ambiguity in natural language. Journal of Artificial Intelligence Research, 11:95-130.

Hansen A. Schwartz and Fernando Gomez. 2008. Acquiring knowledge from the web to be used as selectors for noun sense disambiguation. In CoNLL 2008: Proceedings of the Twelfth Conference on Computational Natural Language Learning, pages 105-112, Manchester, England, August.

Ravi Sinha and Rada Mihalcea. 2007. Unsupervised graph-based word sense disambiguation using measures of word semantic similarity. Irvine, CA, September.

Benjamin Snyder and Martha Palmer. 2004. The English all-words task. In ACL Senseval-3 Workshop, Barcelona, Spain, July. 